\title{
Auto-concepto y desempeño académico en adolescentes. Relaciones con sexo, edad e institución
}

\author{
Sebastián Urquijo ${ }^{2}$
}

\begin{abstract}
Resumen
Este trabajo tuvo como objetivo explorar las relaciones empíricas entre diversas formas de autoconcepto (académico, social, emocional y familiar) y el desempeño académico en lengua y matemáticas de adolescentes que asisten al tercer ciclo de la Educación General Básica, prestando especial atención al sexo, el año y el tipo de institución. Así, se han estudiado 850 alumnos de 7o y 9o años de escuelas públicas y privadas de la ciudad de Mar del Plata, Argentina. Fueron utilizados el Cuestionario de Autoconcepto Forma A (AFA) y pruebas para la Evaluación de la Calidad de la Educación de la Provincia de Buenos Aires. Los resultados indicaron correlaciones estadísticamente significativas entre diversas formas de autoconcepto y el desempeño académico, tanto en lengua cuanto en matemáticas. Sin embargo, los análisis de estas relaciones en función del sexo, el año y el tipo de escuela mostraron que el autoconcepto se asoció al desempeño académico solamente entre los alumnos de las escuelas públicas. Además de eso, para los varones el autoconcepto se asoció al rendimiento en lengua y matemáticas en 7으 y 9으 años, mientras que en las mujeres se encontraron relaciones significativas tan solamente con lengua en 70 año.

Palabras-claves: Autoconcepto; Desempeño académico; Tipo de institución; Género; Edad.
\end{abstract}

\section{Adolescents' self concept and academic achievement. Relationships among gender, age and type of institution}

\begin{abstract}
The aim of this work was to study self-concept (academic, social, emotional and familiar) and academic achievement in language and math exploring sex, grade, and institutional type relations. 850 public and private basic general education students $\left(7^{\text {th }}\right.$ and $9^{\text {th }}$ grades) from Mar del Plata (Argentine) were assessed with the Cuestionario de Autoconcepto Forma A (AFA) and tested in language and math with the Education Quality Assessment of Buenos Aires Government. Statistical analysis showed significant correlations among different forms of selfconcept and academic achievement in language and math. Nevertheless, when considering sex, grade and institution type, the self-concept was associated to academic achievement only in relation to public school students. The male genre presented significant association with self-concept and both language and math in both $7^{\text {th }}$ and $9^{\text {th }}$ grades, although the female gender of $7^{\text {th }}$ grade showed significant relations between language and self-concept.

Keywords: Self-concept; Academic achievement; Type of institution; Gender; Age.
\end{abstract}

\section{Introducción}

Por lo general, cuando hablamos de aprendizaje escolar nos referimos a un complejo proceso en el que intervienen numerosas variables. Algunas de ellas merecen ser destacadas como, por ejemplo, las condiciones psicológicas (afectivas y cognitivas) del niño para acceder al aprendizaje; la relación con el docente; la metodología de la enseñanza, la valoración social del aprendizaje y el medio socio-económico y cultural. La lista de las variables relacionadas al rendimiento académico es muy extensa; encontramos explicaciones que van desde las personales a las no personales y que involucran tanto lo familiar cuanto lo educativo y lo social. En la mayoría de los casos suelen ser mixtas, lo que hace necesario que se tenga que hacer una valoración muy cuidadosa para identificar las causas.

Asimismo, es posible pensar que el éxito escolar consiste en el equilibrio entre el éxito académico, el social y el personal. Esto se consigue mediante el trabajo conjunto de padres, profesores y alumnos en torno a un proyecto común, para

${ }^{1}$ Docente e investigador do Grupo de Psicología Cognitiva y Educacional

${ }^{2}$ Endereço para correspondência:

Facultad de Psicología, Universidad Nacional de Mar del Plata - CONICET - Argentina

Funes 3280 - cuerpo 5 - nivel 3 (7600) Mar del Plata - Argentina.

E-mail: urquijo@mdp.edu.ar 
desarrollar capacidades, hábitos y actitudes (intelectuales, volitivas y sociales). La opinión que los sujetos tengan de su competencia, es fundamental para obtener un buen rendimiento académico. Las experiencias de éxito o fracaso, bajo un juicio de capacidad o incapacidad, crean en el estudiante actitudes que favorecen u obstaculizan el óptimo desarrollo de sus capacidades y potencialidades (Rosenberg, Schooler, Schoenbach, Rosenberg, 1995).

De acuerdo a Musitu, García y Gutiérrez (1997) el auto-concepto puede entenderse como la percepción que el individuo tiene de sí mismo, basado en sus experiencias con los demás y en las atribuciones de su propia conducta. Involucra componentes emocionales, sociales, físicos y académicos. Se trata de una configuración organizada de percepciones de si mismo, admisible a la conciencia y al conocimiento. Es un esquema cognitivo muy complejo construido a través de experiencias previas con respecto al mundo que lo rodea.

El auto-concepto también puede entenderse como la forma en que cada persona se valora y evalúa a sí misma. Existen dos elementos importantes que lo constituyen y son la confianza y la seguridad que las personas tienen en sí mismas. A su vez, la auto-estima puede entenderse como el concepto que tenemos de nuestra valía y se basa en todos los pensamientos, sentimientos, sensaciones y experiencias que, sobre nosotros mismos, hemos ido acumulando durante nuestra vida. El auto-concepto y la auto-estima cumplen un papel importante en los éxitos y los fracasos, la satisfacción, el bienestar psíquico y el conjunto de relaciones sociales. El auto-concepto favorece el sentido de la propia identidad, constituye un marco de referencia para interpretar la realidad externa y las propias experiencias, influye en el rendimiento académico y social, condiciona las expectativas y la motivación, y contribuye a la salud y al equilibrio psíquico. Tener un auto-concepto positivo ayuda a aceptar desafíos, a no tener miedo de desarrollar habilidades, a arriesgarse y a probar cosas nuevas. En cambio, un auto-concepto negativo genera falta de confianza en sí mismo, bajo rendimiento académico y social, una visión distorsionada de sí mismo y de los demás, y una vida personal infeliz (Musitu, García y Gutiérrez, 1997).

El auto-concepto tiene especial importancia desde el punto de vista educativo. Los sujetos con baja auto-estima tienden a desmerecer su talento, son influenciables, eluden situaciones que le provocan ansiedad y se frustran con mayor facilidad. Numerosos autores (Purkey, 1970; Kifer, 1975; Covington y Beery, 1976; Covington y Omelich, 1979; Chapman y Boersma,
1980; Winne, Woodlands y Wong, 1982; Byrne, 1984; Hamachek, 1987; Cooley y Ayres, 1988; Markus, Cross y Wurf, 1990; Leondari, 1993; Abouserie, 1995; Musitu, García y Gutiérrez, 1997; Villarroel Henríquez, 2000) confirman la importancia que tiene el auto-concepto sobre el rendimiento académico.

La literatura científica presenta numerosos trabajos que exploran las relaciones entre el autoconcepto y el rendimiento académico. Trabajos como el de Villarroel Henríquez (2000) apoyan la asociación lineal entre autoconcepto y rendimiento académico, la influencia recíproca entre las expectativas del profesor, el autoconcepto y el rendimiento del alumno, y el efecto que tiene el rendimiento logrado por el alumno sobre la percepción que el profesor tiene de él. Otros trabajos como los de Purkey (1970), Kifer (1975), Covington y Omelich (1979), Byrne (1984), Hamachek (1987), Markus, Cross y Wurf (1990) o Leondari (1993) permiten sustentar la idea de que la cognición y los sentimientos al respecto de si mismo son factores determinantes en el éxito académico de los individuos. Muchos de esos estudios tienen base en los trabajos de Covington y Beery (1976), que consideran que una percepción positiva de si mismo y de las habilidades que cada uno tiene, son esenciales para un buen desempeño escolar y el consecuente logro académico. Algunas investigaciones como las de Chapman y Boersma (1980), Winne, Woodlands y Wong (1982), Cooley y Ayres (1988) o Abouserie (1995) han generado evidencias empíricas de que los niños con dificultades crónicas de aprendizaje tienen un concepto académico de si mismos mas bajo que sus compañeros con desempeño académico normal. También existen algunos estudios que evaluaron el auto-concepto general en niños con dificultades en áreas específicas del conocimiento y encontraron una tendencia a generalizar las imágenes parciales negativas a una imagen negativa general (Black, 1974; Chapman \& Boersma, 1979; Butkowsky \& Willow, 1980). Otros estudios, como los de Rosemberg, Schooler y Schoembach (1989), comprobaron la existencia de una relación causal bidireccional entre auto-estima y depresión asociada a la delincuencia juvenil y a un desempeño académico pobre. Según Luthar y Blatt (1995), los sujetos con alto grado de auto-crítica muestran mayor depresión que se asocia con dificultades en el desempeño académico y el funcionamiento interpersonal.

Los resultados obtenidos por Kifer (1975) han permitido fundamentar la idea de que el éxito en las tareas académicas se encuentra altamente relacionado con características positivas de la personalidad y que el 
fracaso escolar se encuentra estrechamente vinculado a bajos niveles de auto-estima y bajos niveles de capacidad o habilidad. En 1995, Rosemberg, Schooler, Schoenbach y Rosemberg encontraron que la auto-estima académica es un buen predictor del rendimiento escolar.

Sin embargo, como consideramos que el desempeño académico se relaciona con otros factores evaluamos la existencia de diferencias según el sexo. En esa misma dirección, un estudio realizado por Au (1995) sobre el efecto del fracaso académico y las atribuciones causales sobre la desesperanza aprendida de quienes tienen bajo desempeño académico, indicó que los varones tienen más experiencias de fracaso académico y desesperanza aprendida que las mujeres. Por el contrario, los resultados de Rothenberg (1995) sugieren que, para las niñas, los grados medios pueden ser ocasión de una significativa disminución de su autoestima y desempeño académico. Las razones de ello no están claras, pero involucran a varios factores. Uno de ellos, sería el trato preferencial que los varones reciben en la clase. Comparadas con los varones, las adolescentes padecen mayores niveles de estrés, tienen mayor probabilidad de ser depresivas y de cometer suicidio. La depresión en las jóvenes se halla asociada a sentimientos negativos acerca de su cuerpo y apariencia.

En síntesis, la literatura especializada revela un esfuerzo sistemático por explorar y determinar las relaciones entre el auto-concepto y algunos aspectos de la educación. Los trabajos citados se caracterizan por confirmar la existencia de relaciones significativas entre el desempeño escolar y el auto-concepto. Pese a eso, parecería interesante evaluar si estas relaciones se mantienen constantes cuando se consideran variables como edad, sexo, curso y tipo de escuela a la que asisten los alumnos. Este último factor representa variabilidad en el nivel socio-económico del alumno o en las metodologías de la enseñanza, que podrían tener influencias diferentes sobre los procesos de adquisición de conocimientos escolares.

Así, decidimos por estudiar las relaciones entre el auto-concepto y el rendimiento escolar en adolescentes. Al mismo tiempo, explorar las características del auto-concepto en adolescentes y su asociación con la edad, el sexo, el curso y el tipo de escuela a la que asiste. En ese contexto, planteamos la hipótesis de que "los niveles de diferentes formas de auto-concepto en adolescentes se asocian con los niveles del rendimiento académico en lengua y matemáticas, variando de acuerdo al sexo, el curso y el tipo de escuela a la que asisten".

Psico-USF, v. 7, n. 2, p. 211-218, Jul./Dez. 2002

\section{Método}

\section{Participantes}

Se trabajó con 888 alumnos de 70 y 9ㅇ año de escuelas públicas (tres marginales, de bajos recursos/ alto riesgo y tres céntricas, de recursos medios/mediano riesgo) y privadas (tres escuelas céntricas, de altos recursos/bajo riesgo) de la ciudad de Mar del Plata, Argentina. Del total, 447 eran de sexo femenino - 253 de 7o año de EGB y 194 de 9o año de EGB - y 441 de sexo masculino - 247 de 7o año de EGB y 194 de 9o año de EGB. La media de edad del total de la muestra fue de 13,47 años. La media de edad de los alumnos de 7ㅇ año fue de 12,44 años (DS 0,8072) y la de los alumnos de 9o fue de 14,36 años (DS 0,6894). El criterio de selección fue la asistencia a clase el día de administración de los instrumentos de evaluación. Al final, se obtuvieron datos completos de 706 estudiantes.

\section{Instrumentos}

Para evaluar el desempeño académico se utilizaron las pruebas de logro en las áreas de Lengua y Matemática utilizadas en los operativos de la Provincia de Buenos Aires para la evaluación de la calidad educativa. La prueba de matemática constó de 30 ítems, en tanto la evaluación de lengua constó de 35 ítems para el nivel de 7o grado y 39 ítems para 9o grado, diferenciados en tres partes que permitieron conocer el progreso en la escucha, la lectura y la escritura de textos.

Para evaluar el Auto-concepto se utilizó el Tests de Auto-concepto Forma -A (AFA) (Musitu, García y Gutiérrez (1997). Consta de 36 ítems, divididos en 4 factores, que representan diferentes escalas: Autoconcepto Académico (11 ítems), Social (5 ítems), Emocional ( 9 ítems) y Familiar (6 ítems), además de 5 ítems que no participan de ningún factor empírico y si de los factores racionales. Este cuestionario ha sido elaborado a partir de una base de ítems inicial en la que se intentó recoger el universo de definiciones del autoconcepto. Para completar al cuestionario tendrán tres opciones: responderán con " 1 ” si sucede siempre, con " 2 " si sucede algunas veces, y con " 3 " si no sucede nunca. La puntuación directa de cada factor se obtiene asignando 1 (siempre), 2 (a veces) o 3 puntos (nunca), según la respuesta. La suma de las puntuaciones de los cuatro factores, permite obtener la puntuación directa del autoconcepto total.

\section{Resultados}

En la Tabla 1, se presentan las medias obtenidas con cada uno de los instrumentos utilizados, para toda 
la muestra y discriminados según el curso, el sexo y el resultados muestran variabilidad. tipo de escuela a la que concurren. A simple vista esos

Tabla 1 - Medias de las variables estudiadas para toda la muestra y discriminadas por curso, sexo y tipo de escuela

\begin{tabular}{|c|c|c|c|c|c|c|c|c|c|}
\hline & \multirow{3}{*}{$\begin{array}{c}\text { Muestra } \\
\text { Total }\end{array}$} & \multicolumn{4}{|c|}{ 7o año } & \multicolumn{4}{|c|}{ 9o año } \\
\hline & & \multicolumn{2}{|c|}{ Femenino } & \multicolumn{2}{|c|}{ Masculino } & \multicolumn{2}{|c|}{ Femenino } & \multicolumn{2}{|c|}{ Masculino } \\
\hline & & Pub & Pri & Pub & Pri & Pub & Pri & Pub & Pri \\
\hline Desempeño Matemáticas & 11,84 & 10,43 & 15,16 & 9,88 & 15,32 & 9,32 & 18,28 & 10,09 & 18,52 \\
\hline Desempeño Lengua & 16,46 & 12,81 & 20,65 & 10,97 & 17,92 & 16,57 & 24,42 & 16,00 & 24,77 \\
\hline Auto-concepto Académico & 23,95 & 23,97 & 24,50 & 24,00 & 23,75 & 24,31 & 23,42 & 23,65 & 23,93 \\
\hline Auto-concepto Social & 12,59 & 12,20 & 12,69 & 12,58 & 12,88 & 12,68 & 12,26 & 12,70 & 13,21 \\
\hline Auto-concepto Emocional & 18,17 & 17,20 & 17,45 & 18,71 & 18,70 & 17,53 & 17,12 & 19,44 & 19,43 \\
\hline Auto-concepto Familiar & 14,69 & 14,95 & 15,36 & 14,20 & 14,54 & 14,89 & 15,12 & 14,55 & 14,29 \\
\hline Auto-concepto Total & 69,41 & 68,34 & 70,02 & 69,51 & 69,88 & 69,43 & 67,95 & 70,35 & 70,87 \\
\hline
\end{tabular}

Un análisis con la prueba $t$ indicó que el sexo de los alumnos y las medias de las escalas de autoconcepto social $(\mathrm{t}=-2,437)$, emocional $(\mathrm{t}=-7,571)$, familiar $(\mathrm{t}=3,711)$ y total $(\mathrm{t}=-2,275)$ y el desempeño en lengua $(\mathrm{t}=2,391)$ presentan diferencias estadísticamente significativas $(p<0,05)$ mientras que el auto-concepto académico $(\mathrm{t}=0,649)$ y el logro en matemáticas $(t=0,181)$, no las presentan $(p>0,05)$. De la misma forma, el mismo análisis estadístico indicó que las medias del desempeño en Lengua y Matemáticas tienen diferencias estadísticamente significativas $(p=0,000)$ en relación al género (Lengua, $\mathrm{t}=-14,810$ y Matemáticas $\mathrm{t}=-17,121)$ y según el tipo de escuela al que concurren (Público en Lengua $t=13,86$ y en Matemáticas $t=9,99$; Privado en Lengua $t=22,04$ y en Matemáticas $t=16,87$ ).

A continuación, en la Tabla 2, con el objeto de determinar el grado de asociación entre las variables que evalúan el desempeño académico y los factores de autoconcepto, se presentan los resultados del análisis de correlación, utilizando la prueba del producto de los momentos de Pearson, para todos los sujetos.

Tabla 2 - Coeficientes de correlaciones de Pearson de las variables estudiadas para toda la muestra

\begin{tabular}{l|cc}
\hline & Matemáticas & Lengua \\
\hline Auto-concepto Académico & $0,131\left({ }^{* *}\right)$ & $0,101\left({ }^{*}\right)$ \\
Auto-concepto Social & $0,130\left(^{* *}\right)$ & $0,167(* *)$ \\
Auto-concepto Emocional & 0,025 & 0,022 \\
Auto-concepto Familiar & 0,083 & 0,052 \\
Auto-concepto Total & $0,130\left(^{* *}\right)$ & $0,115\left(^{*}\right)$ \\
\hline
\end{tabular}

$(*)$ Nivel de significación $<0,05 \quad(* *)$ Nivel de significación $<0,01$

En estos resultados se puede observar que las variables de auto-concepto emocional y familiar no se asocian de forma estadísticamente significativa con el desempeño académico en lengua o en matemáticas. Sin embargo, se confirman parcialmente los supuestos iniciales derivados de la literatura, por los cuáles, existen asociaciones significativas positivas entre el autoconcepto académico, social y total con el desempeño en matemáticas y lengua.

Sin embargo, tal como proponíamos en la introducción y al haber constatado la existencia de diferencias significativas según el sexo y el tipo de escuela a las que concurren estos alumnos, consideramos importante analizar esas asociaciones, discriminando estas variables. A continuación, en las Tablas 3 y 4, se presentan los resultados del análisis de correlación, utilizando la prueba del producto de los momentos de Pearson, discriminando las variables según el curso, el sexo y el tipo de escuela a la que concurren los alumnos. 
Tabla 3 - Coeficientes de correlaciones de Pearson de las variables estudiadas para los 7o años, por sexo y tipo de escuela

\begin{tabular}{|c|c|c|c|c|c|c|c|c|}
\hline \multirow{3}{*}{$\begin{array}{r}\text { Sexo } \\
\text { Escuela } \\
\text { Área }\end{array}$} & \multicolumn{4}{|c|}{ Femenino } & \multicolumn{4}{|c|}{ Masculino } \\
\hline & \multicolumn{2}{|c|}{ Pública } & \multicolumn{2}{|c|}{ Privada } & \multicolumn{2}{|c|}{ Pública } & \multicolumn{2}{|c|}{ Privada } \\
\hline & Matem & Lengua & Matem & Lengua & Matem & Lengua & Matem & Lengua \\
\hline Auto-concepto Académico & 0,014 & $0,222 *$ & 0,186 & $-0,052$ & $0,416^{* *}$ & $0,257 *$ & 0,168 & 0,316 \\
\hline Auto-concepto Social & 0,046 & $0,231 *$ & $-0,234$ & $-0,115$ & $0,339 * *$ & $0,346 * *$ & $-0,224$ & 0,153 \\
\hline Auto-concepto Emocional & 0,043 & 0,126 & 0,096 & $-0,076$ & 0,080 & 0,075 & $-0,173$ & 0,138 \\
\hline Auto-concepto Familiar & $-0,034$ & 0,138 & $-0,097$ & $-0,125$ & $0,424 * *$ & $0,341 * *$ & $-0,010$ & 0,197 \\
\hline Auto-concepto Total & 0,025 & $0,253 *$ & 0,030 & $-0,128$ & $0,449 * *$ & $0,344^{* *}$ & $-0,061$ & 0,316 \\
\hline
\end{tabular}

(*) Nivel de significación $<0,05-(* *)$ Nivel de significación $<0,01$

Tabla 4 - Coeficientes de correlaciones de Pearson de las variables estudiadas para los 9 años, por sexo y tipo de escuela

\begin{tabular}{r|cccccccc} 
Sexo & \multicolumn{4}{r}{ Femenino } & \multicolumn{4}{c}{ Masculino } \\
\cline { 2 - 9 } Tipo Escuela & \multicolumn{2}{|c}{ Pública } & \multicolumn{2}{c}{ Privada } & \multicolumn{2}{c}{ Pública } & \multicolumn{2}{c}{ Privada } \\
Área & Matem & Lengua & Matem & Lengua & Matem & Lengua & Matem & Lengua \\
Auto-concepto Académico & 0,158 & 0,145 & 0,092 & $-0,075$ & $0,292\left(^{*}\right)$ & $0,271\left(^{*}\right)$ & 0,138 & 0,010 \\
Auto-concepto Social & 0,070 & 0,081 & 0,265 & 0,015 & $-0,005$ & 0,202 & 0,237 & 0,196 \\
Auto-concepto Emocional & $-0,021$ & $-0,093$ & 0,212 & 0,064 & 0,004 & 0,083 & $-0,043$ & $-0,133$ \\
Auto-concepto Familiar & $-0,020$ & $-0,100$ & $-0,052$ & $-0,069$ & 0,203 & 0,038 & 0,044 & $-0,083$ \\
Auto-concepto Total & 0,063 & 0,014 & 0,167 & $-0,031$ & $0,234\left(^{*}\right)$ & 0,250 & 0,152 & 0,007 \\
\hline
\end{tabular}

(*) Nivel de significación $<0,05-(* *)$ Nivel de significación $<0,01$

Estos resultados resultan sumamente interesantes, desde el momento en que muestran que, en todos los casos, las asociaciones entre desempeño académico y diferentes formas de auto-concepto varían significativamente en función de curso, el sexo y el tipo escuela al que asisten los alumnos. En los alumnos de las escuelas privadas, independientemente del sexo o la edad, no encontramos ninguna relación entre el desempeño académico en lengua o en matemáticas. En las escuelas públicas, también se observa otro hecho interesante y es que en las mujeres, sólo en el 7으 año, su auto-concepto se asocia al desempeño en el área de lengua. Para los alumnos varones de escuelas públicas la relación de su desempeño, tanto en lengua cuanto en matemáticas, se asocia sistemáticamente al autoconcepto, aunque debe destacarse que estas asociaciones disminuyen y se diluyen con el tiempo y son más fuertes en los sujetos de los cursos inferiores.

\section{Discusión y Consideraciones Finales}

Los resultados permiten confirmar la hipótesis de este trabajo, es decir que "los niveles de diferentes formas de auto-concepto en adolescentes se asocian con los niveles del rendimiento académico en lengua y matemáticas, variando de acuerdo al sexo, el curso y el tipo de escuela a la que asisten”. Los análisis estadísticos permitieron establecer que existen asociaciones estadísticamente significativas entre el auto-concepto total y el desempeño académico en términos generales. Si bien los niveles de correlación pueden caracterizarse como bajos, son estadísticamente significativos $\mathrm{y}$, por ello, podemos hablar de la existencia de una tendencia general que indicaría que a medida que aumenta el autoconcepto total de un sujeto, aumenta el desempeño académico en lengua y en matemáticas. Esta tendencia, caracterizada por asociaciones débiles, indicaría que no se trata de las únicas variables que intervienen en el desempeño académico. Por supuesto, el logro escolar es un fenómeno complejo, determinado por innumerables variables. Sin embargo, los resultados permiten sostener la idea de que el auto-concepto incide sobre él. Estos resultados coinciden con lo expuesto en la literatura científica, ya que los sentimientos negativos acerca de uno mismo, como también la sensación de incapacidad y fracaso, influirían negativamente en el logro escolar (Purkey, 1970; Kifer, 1975; Covington y Beery, 1976; Covington y Omelich, 1979; Chapman y Boersma, 1980; Winne, Woodlands y Wong, 1982; Byrne, 1984; Hamachek, 1987; Cooley y Ayres, 1988; Markus, Cross y Wurf, 1990; Leondari, 1993; Abouserie, 1995; Musitu, García y Gutiérrez, 1997; Villarroel Henríquez, 2000). 
Sin embargo, no todas las escalas de autoconcepto presentaron asociaciones con el desempeño. Solamente las de auto-concepto académico y social. Las relaciones con el auto-concepto familiar y el emocional no fueron estadísticamente significativas. Resulta evidente que el auto-concepto académico se encuentre relacionado al desempeño académico, ya que la percepción que uno tiene de si mismo sobre sus habilidades y capacidades para el estudio, ineludiblemente debería influir sobre el logro. Un alumno que se considera poco hábil, probablemente se esfuerce poco para mejorar y no sienta presiones para obtener buenas calificaciones.

Independientemente de este resultado general, coherente con la literatura (Au, 1995; Rothenberg, 1995), los niveles de auto-concepto resultaron significativamente diferentes según el sexo, el curso y el tipo de escuela al que concurrían los sujetos. Sorprendentemente, ya que no encontramos antecedentes semejantes en la literatura, las asociaciones significativas entre autoconcepto y desempeño académico, se observan solamente en los alumnos de las escuelas públicas y presentan variaciones de acuerdo al sexo y al curso al que asisten. Así, se destacan especialmente en los alumnos varones y son más consistentes en el séptimo año. En las mujeres del 7o año, el auto-concepto total, académico y social se relaciona con el desempeño en el área de lengua, en tanto que en los hombres, tanto de 7 ㅇ cuanto de 9o año, se relaciona con el desempeño en ambas áreas.

Estos resultados deberían alertar a los profesionales de la educación sobre los riesgos de realizar generalizaciones amplias que llevan a suponer que el auto-concepto se encuentra asociado al desempeño académico de forma generalizada. Hemos presentado evidencias que sugieren que solo se verifica en alumnos de escuelas públicas y que, por lo tanto, encontraremos alumnos de escuelas privadas que tengan un bajo auto-concepto y, sin embargo, presenten un rendimiento académico normal o superior. También debería tenerse mucho cuidado al suponer que un mejoramiento de la auto-estima podría beneficiar los aprendizajes, sin considerar el sexo, la edad o el nivel socio-económico y cultural de los individuos. Los resultados reconfirman que el aprendizaje de contenidos escolares es un proceso sumamente complejo, que depende de innumerables factores que covarían entre si.

Resta proponer alguna explicación para las diferencias observadas en el auto-concepto y el desempeño, de acuerdo al tipo de escuela al que asisten, al curso y el sexo de los alumnos.
Con relación al primer problema, las diferencias socio-económicas y culturales que caracterizan a los alumnos de escuelas públicas y privadas, sumadas a las diferencias en metodología de enseñanza y a la presión académica (cantidad y calidad de exigencias académicas y el apoyo escolar), la infraestructura, el acceso a la tecnología, la cobertura de las necesidades básicas y la estimulación intelectual en el hogar, permitirían explicar por que los alumnos de las escuelas privadas presentan un nivel de logro significativamente superior. A su vez, la presencia de niveles promedio superiores en el autoconcepto, podrían relacionarse al status socioeconómico y cultural de los sujetos. El apoyo familiar, el status que representa la asistencia a escuelas consideradas socialmente como de excelencia, la asistencia personalizada, la preocupación por la resolución de los problemas académicos y personales de los alumnos a través de gabinetes psicopedagógicos, podrían ser factores de peso en la explicación de que se observen niveles superiores de auto-concepto. En contraposición, en las escuelas públicas se observa, con mayor frecuencia, la presencia de alumnos desfasados en la edad, por repetición o deserción, factores que influyen en la auto-estima y en el desempeño. Muchas veces esos niños deben cumplir con otras actividades relacionadas al sustento económico (trabajando o mendigando), a la ayuda en el hogar (cuidando a hermanos o realizando tareas domésticas) o se encuentran expuestos a abusos y malos tratos. Esto, sin duda, produce un importante impacto académico y psicológico, ya que no disponen de tiempo o motivación para el estudio, aumentan sus síntomas individual, asociada depresivos y disminuyen su auto-concepto. Esta situación a las características de las escuelas públicas que atienden poblaciones desfavorecidas y que deben preocuparse por la contención emocional, la satisfacción de necesidades básicas (alimentación y vestido) y la formación de hábitos básicos, incidiría significativamente en el desempeño académico.

Los alumnos de las escuelas privadas presentan, sistemáticamente, un nivel superior de auto-concepto y rendimiento académico. Pero, en ese caso, los niveles de logro académico no pueden explicarse por el autoconcepto, ya que las asociaciones no resultaron estadísticamente significativas. Probablemente, el medio familiar, asociado a las características de la institución escolar a la que asisten, determinan características económicas y culturales que pueden limitar o favorecer su desarrollo personal y educativo. Los resultados permitirían sustentar la idea de que existe una estrecha unión entre el éxito académico y el origen social. Desde 
la perspectiva del aprendizaje, los niños de clases sociales más elevadas pueden interiorizar pautas y conductas académicas relevantes. Con esto no quiero decir que los alumnos de las escuelas públicas no puedan hacerlo, sino que para el alumno de escuelas privadas es más sencillo y están más disponibles. El ambiente cultural que los progenitores ofrecen a sus hijos ejerce una poderosa influencia en el proceso de desarrollo de la personalidad, la inteligencia y la socialización. La situación social, cultural y familiar del alumno, resulta determinante del riesgo al fracaso.

Con relación a las diferencias que se observan en las escuelas públicas, en función del curso y el sexo de los alumnos, encontramos estudios (Au, 1995; Rothenberg, 1995), que hacen referencia a que la autoestima se asocia al sexo y a la edad cronológica de los sujetos, indicando que los varones tenían más experiencias de fracaso académico y desesperanza aprendida que las mujeres, y que para las niñas, los grados medios pueden ser ocasión de una significativa disminución de la autoestima y el desempeño académico. No obstante, a medida que aumenta la edad, es natural esperar un aumento de la auto-estima, por ello, resulta esperable observar que los alumnos de 7으 año presenten medias inferiores a las de los alumnos de 9․

Para concluir este trabajo, quisiéramos destacar que la realidad educativa no se puede desligar de su componente humano, ya que en el hecho de planificar la enseñanza se debe tener en cuenta que existen seres humanos diferentes. Y no sólo por sus características personales, sino también por los distintos condicionantes sociales. No se puede ignorar la estrecha relación que existe entre buenos resultados académicos y procedencia social. También, la mayoría de los alumnos de las escuelas privadas poseen recursos sociales y económicos que favorecen su auto-estima. Por ello, debemos prestar especial atención y cuidados a los alumnos que asisten a escuelas desfavorecidas, ya que en ellos las características socio-afectivas tienen mayor impacto en el aprendizaje escolar.

\section{Referencias}

Abouserie, R. (1995). Self-esteem and achievement motivation as determinants of student's approaches to studying. Studies in Higher Education, 20(1), p. 19-26.

Au, R. C. P. (1995). Academic failure and learned hopelessness in Hong Kong academically low achievers. Bulletin of the Hong Kong Psychological Society, 34(35), p. 83-100.
Black, W. F. (1974). Self concept as related to achievement and age in learning-disabled children. Child Development, 35. p. 1137-1140.

Butkowsky, I. S. \& Willow, D. M. (1980). Cognitive motivational characteristics of children varying in reading ability: evidence of learned helplessness in poor readers. Journal of Educational Psychology, 72(3), p. 408-422.

Byrne, B. M. (1984). The general/academic self-concept nomological network: a review of construct validation research. Review of Educational Research, 54, p. 427-456.

Chapman, J. \& Boersma, F. J. (1979). Academic self concept in elementary learning disabled children. A study with the student's perception of ability scale. Psychology in the Schools, 16(2), p. 201-206.

Chapman, J. \& Boersma, F. J. (1980). Affective Correlates of Learning Disabilities. New York: Lisse, Swets \& Zeitlinger.

Cooley, E. J. \& Ayres, R. R. (1988). Self concept and success-failure attributions of nonhandicapped students and students with learning disabilities. Journal of Learning Disabilities, 21, p. 174-178.

Covington, M. V. \& Beery, R. G. (1976). Self Worth and SchoolLearning. New York: Holt, Rinehart \& Winston.

Covington, M. V. \& Omelich, C. L. (1979). Are causal attributions causal? A path analysis of the cognitive model of achievement motivation. Journal of Personality and Social Psychology, 37, p. 1487-1504.

Hamachek, D. E. (1987). Encounters with the self. New York: Holt, Rinehart \& Winston.

Kifer, E. (1975). Relationships between academic achievement aand personality characteristics: a quasilongitudinal study. American Educational Research Journal, 12(2), p. 191-210.

Leondari, A. (1993). Comparability of self-concept among normal achievers, low achievers and children with learning difficulties. Educational Studies, 19(3), p. 357371.

Luthar, S. \& Blatt, S. J. (1995). Differential vulnerability of dependency and self-criticism among disadvantaged teenagers. Journal of Research on Adolescence; 5(4), p. 431-449.

Markus, H., Cross, S. \& Wurf, E. (1990). The role of the self system in competence. In Sternberg, J. \& Kolligian, J. Jr. (Eds.). Competence Considered. New Haven: Yale University Press. 
Musitu, G., García, F. \& Gutiérrez, M. (1997). Autoconcepto Forma A (A.F.A). Manual. Madrid. TEA Ediciones.

Purkey, W. W. (1970). Self Concept and School Achievement. Englewood Cliffs, NJ: Prentice Hall.

Rosenberg, M., Schooler, C. \& Schoenbach, C. (1989). Self-esteem and adolescent problems: modeling reciprocal effects. American Sociological Review; 54(6), p. 1004-1018.

Rosenberg, M., Schooler, C., Schoenbach, C. \& Rosenberg, F. (1995) Global self-esteem and specific self-esteem: different concepts, different outcomes. American Sociological Review; 60(1), p. 141-156.

Rothenberg, D. (1995). Supporting Girls in Early Adolescence. ERIC Digest. ERIC Clearinghouse on Elementary and Early Childhood Education, Urbana, III.
Sisto, F., Urquijo, S. \& Souza, M. T. (1999). Peer acceptance and cognitive development. Psychological Reports, 84(2), p. 611-616.

Villarroel Henríquez, V. A. (2000). Relación entre Autoconcepto y Rendimiento Académico. Universidad Católica de Chile. Revista Psykhe. 10(1).

Waserman, M. (2000). Fracaso Escolar. Revista de Actualidad Psicológica. 15(252), p. 7-11.

Winne, P. H., Woodlands, M. J. \& Wong, B. Y. L. (1982). Comparability of self concept among learning disabled, normal and gifted students. Journal of Learning Disabilities, 15, p. 470-475.

Recebido em 30/08/2002 Reformulado em 07/11/2002 Aceito em 10/12/2002

Sobre o autor:

Sebastián Urquijo é licenciado em Psicologia, mestre e doutor pela Faculdade de Educação da Universidade Estadual de Campinas, e professor de Psicologia Cognitiva e Teorias da Aprendizagem, na Faculdade de Psicologia da Universidade Nacional de Mar del Plata, Argentina. 